\title{
Análisis retrospectivo de 23 años de necrólisis epidérmica tóxica en la Unidaad de Quemados de Alicante, España
}

\author{
Retrospective analysis of 23 years of toxic epidermal necrolysis \\ in the Burn Unit of Alicante, Spain
}

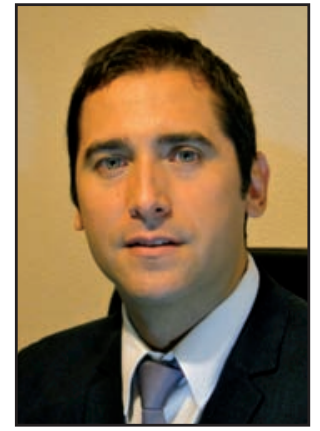

Castillo-Muñoz, F.I.

Castillo-Muñoz, F.I. ${ }^{*}$ Céspedes-Guirao, F.J.*, Novo-Torres, A. ${ }^{\star \star}$, Lorda-Barraguer, E.***

Resumen

El Síndrome de Lyell o Necrólisis Epidérmica Tóxica (NET) se encuadra dentro del espectro de reacciones cutáneas adversas graves y se caracteriza por presentar una baja incidencia pero una elevada mortalidad. La mayoría de los casos de NET se relacionan con una reacción medicamentosa idiosincrática.

El objetivo de este trabajo es reflejar la experiencia clínica con esta entidad en la Unidad de Quemados del Hospital General Universitario de Alicante, España, durante los últimos 23 años.

Realizamos un estudio retrospectivo recogiendo los datos epidemiológicos, agentes causales probables, pruebas complementarias, patologías concomitantes, tratamiento y evolución clínica. En total recogimos 16 pacientes con una media de edad de 53,19 años, $68,75 \%$ mujeres y $31,25 \%$ varones. Los antibióticos fueron los fármacos más comúnmente implicados $(31,25 \%)$, seguidos de los AINES (25\%). Durante el ingreso hospitalario, el 43,75\% de los pacientes recibió corticoides sistémicos, el 37,5\% exclusivamente tratamiento de soporte y el $25 \%$ inmunoglobulinas intravenosas. El 56,25\% sufrió algún tipo de secuela, siendo la afectación ocular la más frecuente (18,75\%).

Calculamos el SCORTEN (Severity of illness score for toxic epidermal necrolisys) para todos los pacientes en las primeras 24 horas de ingreso, siendo la suma de las probabilidades predichas de morir por NET de un $32 \%$, cifra superior a los datos reales (mortalidad del $25 \%$ ).

La utilización de corticoides sistémicos sigue siendo controvertida, apareciendo en nuestro estudio un mayor número de infecciones y una mayor mortalidad asociada a dicho tratamiento. La retirada inmediata del fármaco sospechoso, el ingreso precoz en una Unidad de Quemados o de Cuidados Intensivos, el tratamiento de soporte y la reposición hidroelectrolítica, siguen siendo medidas cruciales y de primera línea en el tratamiento de estos pacientes.

A pesar de que están descritos diferentes tipos de tratamiento farmacológico para el abordaje de la NET, ninguno ha logrado un rol convincente, por lo que consideramos que se necesitan más estudios prospectivos aleatorizados multicéntricos.

Proponemos en este artículo un protocolo de actuación ante este tipo de pacientes.

\begin{tabular}{|l} 
Palabras clave $\begin{array}{l}\text { Síndrome de Lyell, Necrólisis epidérmica tóxica, } \\
\text { Reacciones medicamentosas adversas, } \\
\text { Reacciones cutáneas adversas. }\end{array}$ \\
Nivel de evidencia científica $\quad$ IV
\end{tabular}

Key words Lyells syndrome, Toxic epidermal necrolysis, Adverse drug reactions, Adverse cutaneous reactions.

Level of evidence
Abstract

Lyell's syndrome or Toxic Epidermal Necrolysis (TEN) is considered within the spectrum of severe cutaneous adverse reactions; it's a rare disease but with a high mortality rate. Most of the cases are related to idiosyncratic drug reactions.

The aim of this paper is to reflect the clinical experience with this pathology at the Burn Unit of the Universitary General Hospital of Alicante, Spain, for the past 23 years.

We conduct a retrospective study, collecting epidemiological data, likely causative agents, additional tests, comorbidities, treatment and clinical evolution, including 16 patients with an average age of 53,19 years; $68,75 \%$ were females and $31,25 \%$ were males. Antibiotics were most commonly implicated drugs (31.25\%), followed by nonsteroidal anti-inflammatory drugs (25\%). During admission, $43,75 \%$ received systemic corticosteroids, $37,5 \%$ only supportive treatment and 25\% intravenous immunoglobulin; $56.25 \%$ suffered some type of sequel, being the most frequent ocular involvement $(18,75 \%)$.

The SCORTEN (Severity of illness score for toxic epidermal necrolisys) was calculated for all patients in the first 24 hours of admission, the sum of the predicted probabilities of dying from NET it's $32 \%$, higher than the actual data (mortality $25 \%$ ).

The use of systemic corticosteroids remains controversial, appearing in our study a greater number of infections and increased mortality associated with this treatment. Early admission in Burn or Intensive Care Unit, the prompt withdrawal of suspected drug, supportive treatment and fluid and electrolyte replacement, are considered crucial and first-line treatment in these patients.

Different drug treatments have been described for the management of this condition without achieving a convincing role, so we considerer that new multicenter randomized prospective studies are required in the future.

We propose in this paper a protocol for managing these patients. 


\section{Introducción}

El síndrome de Lyell o Necrólisis Epidérmica Tóxica (NET), es una enfermedad poco frecuente pero potencialmente mortal. Alan Lyell, dermatólogo de origen escocés, fue quien primero utilizó el término en el año 1956 para describir un raro trastorno mucocutáneo de rápida evolución caracterizado por desepidermización cutánea extensa, eritema y elevado riesgo vital.

Esta entidad se encuadra dentro del espectro de reacciones cutáneas adversas graves debido al intenso desprendimiento de la piel que produce. Bastuji-Garin et al. (1) propusieron la definición más ampliamente aceptada para diferenciar la NET de otra entidad del mismo espectro como es el Síndrome de Stevens-Johnson (SSJ), basándose en la extensión máxima del despegamiento epidérmico. De esta manera, esta superficie es menor del $10 \%$ en el SSJ, del 10 al 30\% en el Síndrome de superposición (SSJ-NET) y mayor del $30 \%$ en la NET.

Encontramos en la bibliografía al respecto incidencias de esta patología de entre 0,4 y 1,2 por millón de habitantes/año (2). Esta incidencia se eleva con la edad (mayor exposición a fármacos) y es al menos 1.000 veces mayor en pacientes VIH positivos o con Síndrome de inmunodeficiencia adquirida (SIDA). Parece que las mujeres se ven afectadas más frecuentemente que los hombres, con una proporción de 1,5:1. La mayoría de los casos de SSJ y NET son secundarios a fármacos, pudiendo afectar potencialmente a cualquier individuo al que se le administren. Están implicados más de 220 fármacos como agentes desencadenantes de este tipo de síndromes, entre los que los más frecuentes son: antibióticos, anticonvulsionantes, antiinflamatorios no esteroideos (AINES) y el alopurinol. En el caso de pacientes VIH/SIDA, el más frecuentemente implicado es la nevirapina, un inhibidor no nucleósido de la transcriptasa inversa. Otras causas posibles son: infecciones, vacunas, productos químicos y radioterapia.

Aunque la NET es un síndrome raro, tiene un impacto significativo en la salud pública debido a su alta morbimortalidad. La mortalidad del SSJ está normalmente por debajo del 5\%, entre el 10 y el $15 \%$ en los casos de SSJNET, mientras que en la NET es de hasta el 33-35\%.

La escala SCORTEN (Severity of illness score for toxic epidermal necrolisys) (3) está específicamente validada para evaluar la severidad de los pacientes con NET o SSJ-NET, y permite una predicción de la mortalidad por esta entidad mediante el análisis de 7 factores de riesgo independientes como son: la edad >40 años; un porcentaje inicial de desepidermización mayor del 10\%; una urea sérica $>28 \mathrm{mg} / \mathrm{dl}$; glucemia $>252 \mathrm{mg} / \mathrm{dl}$; bicarbonato sérico $<20 \mathrm{mEq} / 1$; frecuencia cardiaca $>120$ latidos/minuto y presencia asociada de cáncer o neoplasia hematológica.

En cuanto a la inmunopatogénesis, los últimos estudios hablan de que se trataría de una enfermedad mediada por células T CD8+ que actúan como el principal mediador de la muerte de los queratinocitos. Surgen dos teorías para explicar la activación de estas células: la interacción farmacológica de los fármacos con el sistema inmune del paciente y la teoría pro-hapteno.

El objetivo de este trabajo es analizar la evolución clínica de una serie de 16 pacientes diagnosticados de SSJNET o NET ingresados y tratados en nuestra Unidad de Quemados (UQ) del Hospital Universitario de Alicante, España, durante los últimos 23 años, presentando los aspectos epidemo-etiológicos más importantes, la evolución microbiológica-infecciosa, los tratamientos utilizados, así como los resultados clínicos y secuelas sufridas por los pacientes. Además, realizamos una revisión bibliográfica rigurosa del tema centrada en tres aspectos básicos: clasificación clínica, etiopatogenia y tratamiento farmacológico de la enfermedad, para acabar proponiendo un protocolo de recepción $\mathrm{y}$ tratamiento de estos pacientes.

\section{Material y método}

Llevamos a cabo una búsqueda retrospectiva de todos los casos de NET ingresados a cargo de la Unidad de Quemados del Hospital General Universitario de Alicante (UQ-HGUA) entre enero de 1990 y diciembre de 2013. Recogimos de forma sistemática los siguientes datos a partir de la historia clínica de cada paciente: diagnóstico, edad y sexo, fármaco más probablemente implicado en cada caso, enfermedades de base, descripción de la clínica sistémica, afectación de mucosas, tipo y localización de las lesiones cutáneas, intervalo entre el consumo inicial del fármaco y la aparición de los síntomas, pruebas complementarias realizadas (analítica, biopsia, cultivos microbiológicos), mortalidad, opciones terapéuticas farmacológicas y quirúrgicas empleadas, evolución clínica, días de estancia hospitalaria, puntuación en la escala SCORTEN y presencia de secuelas.

Realizamos la adscripción etiológica del fármaco en función del tiempo transcurrido desde su administración a la aparición del cuadro clínico. Así, consideramos como sospechosos todos los fármacos administrados al paciente entre unos días y 8 semanas antes de la aparición del cuadro y descartamos los fármacos que el paciente ya consumía desde hacía meses o años.

\section{RESULTADOS}

Resumimos los datos obtenidos en las Tablas I-IV, Fig. 1 y 2 y en los Gráficos 1-4. Los pacientes estaban en un intervalo de edad de entre 6 a 88 años, con una media de 53,19 años, siendo más del $80 \%$ mayores de 30 años. El $68,75 \%$ eran mujeres y el $31,25 \%$ varones.

\section{Incidencia}

La incidencia registrada en nuestro hospital fue de 0,43 casos/millón/año de media en los últimos 23 años. Como apreciamos en la Gráfico 1, conforme aumenta 
Tabla I. Características de los pacientes $(\mathrm{N}=16)$

\begin{tabular}{|c|c|c|c|c|c|c|c|}
\hline $\mathbf{N}^{\mathbf{o}}$ & $\begin{array}{l}\text { Sexo } \\
/ \text { Edad }\end{array}$ & Causa & $\begin{array}{c}\text { Días ingreso } \\
\text { en } \\
\text { UQ-HGUA }\end{array}$ & $\begin{array}{c}\text { Intervalo } \\
\text { entre toma y } \\
\text { desarrollo } \\
\text { síntomas }\end{array}$ & \begin{tabular}{|c|} 
Días desde \\
comienzo \\
síntomas \\
hasta ingreso \\
en UQ-HGUA
\end{tabular} & Año & Comorbilidades \\
\hline 1 & $\mathrm{~F} / 6$ & Amoxicilina & 45 & 1 & 2 & 1992 & No \\
\hline 2 & $\mathrm{~F} / 56$ & Ceftibuteno, Ciprofloxacino & 12 & 7 & 5 & 1996 & DM tipo II \\
\hline 3 & $\mathrm{~F} / 88$ & Ceftriaxona, Ofloxacino & 14 & 1 & 5 & 2000 & $\begin{array}{l}\text { DM tipo II, DLP, } \\
\text { Hiperuricemia, HTA }\end{array}$ \\
\hline 4 & $\mathrm{~F} / 21$ & Ibuprofeno & 30 & 1 & 4 & 2001 & RAM a codeína \\
\hline 5 & $F / 31$ & Diclofenaco, Dexketoprofeno & 17 & 7 & 12 & 2001 & RAM a AINES \\
\hline 6 & $\mathrm{M} / 48$ & Desconocido & 18 & 1 & 3 & 2002 & $\begin{array}{l}\text { DM tipo II, Enolismo } \\
\text { crónico, HT portal, EPOC }\end{array}$ \\
\hline 7 & $\mathrm{M} / 78$ & Aceclofenaco, Metamizol & 13 & 2 & 7 & 2006 & $\begin{array}{l}\text { HTA, Anemia crónica, } \\
\text { Apnea del sueño }\end{array}$ \\
\hline 8 & $\mathrm{M} / 60$ & Fenitoína & 14 & 30 & 6 & 2007 & $\begin{array}{l}\text { HTA, Astrocitoma grado } \\
\text { III (operado } 2 \text { meses antes) }\end{array}$ \\
\hline 9 & $\mathrm{~F} / 71$ & $\begin{array}{l}\text { Pantoprazol, Deflazacort, } \\
\text { Colecalciferol, Enalapril }\end{array}$ & 13 & Desconocido & 7 & 2007 & $\begin{array}{l}\text { IRC,Arteritis temporal, } \\
\text { Asma bronquial, } \\
\text { Meningioma (15 años antes) }\end{array}$ \\
\hline 10 & $\mathrm{~F} / 33$ & Desconocido & 20 & Desconocido & 2 & 2008 & $\begin{array}{l}\text { LES con nefropatía } \\
\text { lúpica, HT pulmonar, } \\
\text { Esteatosis hepática }\end{array}$ \\
\hline 11 & $\mathrm{~F} / 71$ & Sulfametoxazol/trimetoprima & 31 & 8 & 4 & 2009 & $\begin{array}{l}\text { HTA, Ca. Mama } \\
\text { (10 años antes) }\end{array}$ \\
\hline 12 & $\mathrm{~F} / 7$ & Ibuprofeno & 24 & 3 & 4 & 2009 & No \\
\hline 13 & $\mathrm{M} / 76$ & Fenitoína & 21 & 10 & 10 & 2010 & $\begin{array}{l}\text { HTA, Obesidad, } \\
\text { Crisis convulsivas } \\
\text { (en tto. con fenitoína) }\end{array}$ \\
\hline 14 & $\mathrm{~F} / 71$ & Alopurinol & 16 & 21 & 21 & 2010 & $\begin{array}{l}\text { HTA, Cardiopatia } \\
\text { isquemica }\end{array}$ \\
\hline 15 & $\mathrm{~F} / 50$ & Penicilina & 1 & 60 & 4 & 2010 & HTA \\
\hline 16 & $\mathrm{M} / 81$ & Alopurinol & 1 & Desconocido & 3 & 2013 & $\begin{array}{l}\text { HTA, DM tipo II, DLP, } \\
\text { Insuf. cardiaca, IRC, } \\
\text { Anemia ferropénica, } \\
\text { Hiperuricemia, } \\
\text { Gastritis atrófica }\end{array}$ \\
\hline
\end{tabular}

IG: inmunoglobulina; iv: intravenoso; vo: vía oral; DM: diabetes mellitus; DLP: dislipemia; HT: hipertensión; ES: lupus eritematoso sistémico; HTA: hipertensión arterial, RAM: reacción alérgica a medicamento; Ca: cáncer; IRC: insuficiencia renal crónica.

anualmente la población de la cual es referencia nuestra UQ-HGUA vemos un aumento proporcional de la incidencia de casos de NET.

\section{Etiologías}

Sólo en 2 pacientes no encontramos una causa farmacológica clara (Tabla I). En el resto hallamos diversos fármacos como posibles agentes causantes de la enfermedad. De entre ellos, los más comúnmente implicados fueron los antibióticos $(31,25 \%)$, seguidos de los AINES
(25\%), los anticonvulsivantes $(12,5 \%)$, el alopurinol $(12,5 \%)$ y otros $(6,25 \%)$. En el $50 \%$ de los pacientes la NET pudo atribuirse a un sólo fármaco y en 6 casos se vieron implicados varios fármacos.

La fenitoína fue el único anticovulsivante implicado. Los $\beta$-lactámicos fueron la causa más frecuente entre los antibióticos (4 pacientes), seguidos de las quinolonas ( 2 pacientes) y las sulfonamidas ( 1 paciente). Los $\beta$-lactámicos implicados fueron la amoxicilina, ceftibuteno, ceftriaxona y penicilina. 
Entre los fármacos que provocaron fallecimiento, en 2 casos fueron antibióticos (ceftriaxona, ofloxacino y penicilina), en 1 caso el alopurinol y en 1 caso el fármaco fue desconocido. Destacan los pacientes 15 y 16 de la serie que fueron tratados con penicilina y alopurinol, respectivamente, porque tuvieron una evolución de la enfermedad muy rápida y agresiva.

Intervalo entre el primer consumo del fármaco, la aparición de los síntomas y el ingreso en la UQ-HGUA

El tiempo medio transcurrido entre la ingestión del fármaco y el inicio de los síntomas fue de 11,7 días, con un intervalo de entre 1 y 60 días. En 3 pacientes no constaban los datos concretos al respecto. En más de la mitad $(56,25 \%)$ los síntomas se desarrollaron dentro de los 9 primeros días. La fenitoína, la penicilina y el alopurinol ofrecieron los intervalos más largos entre el momento de la ingestión y la aparición de los síntomas. La media de

Gráfico 1. Casos de NET ingresados anualmente en la UQ-HGUA durante los últimos 23 años, comparados con la variación anual de la población (en millón de habitantes) de la provincia de Alicante (España) de la cual es referencia.

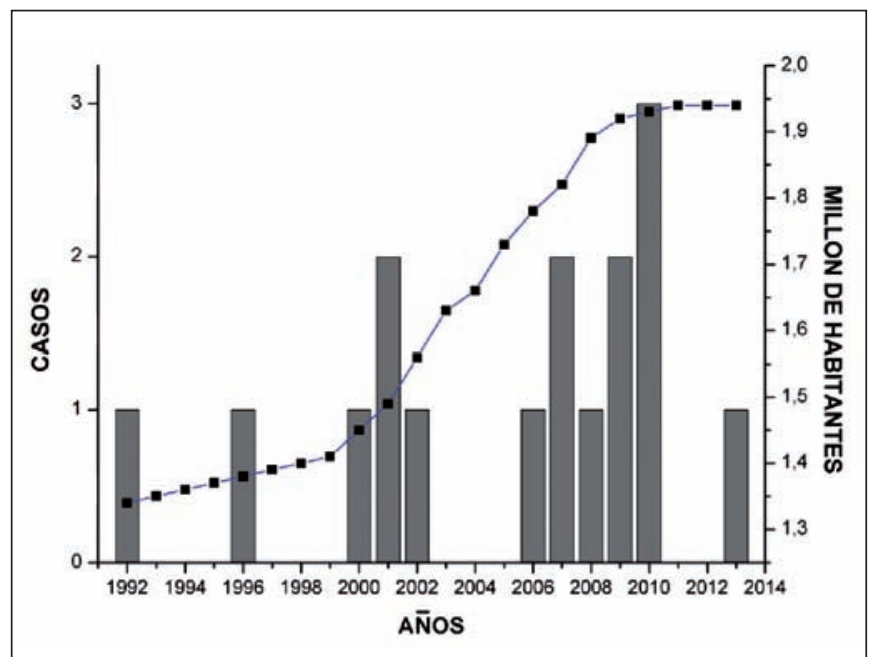

días entre la aparición de síntomas y el ingreso en la UQHGUA fue de 6,18 días.

\section{Comorbilidades}

Excepto los pacientes 1 y 12, ambos menores de 10 años de edad, el resto tenían alguna comorbilidad asociada de entre las que la más común fue la hipertensión arterial $(37,5 \%)$ seguida de la diabetes mellitus (25\%). Hubo 2 pacientes con reacción alérgica conocida a medicamentos (RAM), destacando el paciente 5, que a pesar de presentar RAM a AINES, fue precisamente la toma de estos fármacos la que causó el cuadro de NET. Sólo en el paciente 8 existía coexistencia de neoplasia activa y NET.

\section{Tratamiento previo al ingreso en la Unidad de Quemados}

Destacamos que todos los pacientes del grupo de estudio habían sido tratados previamente al ingreso en la UQ-HGUA en otro centro médico o en otro Servicio de nuestro hospital (Tabla II). El 68,75\% con corticoides sistémicos, el 47,75\% con antihistamínicos sistémicos, el $25 \%$ con antibióticos y el 12,5\% con corticoides tópicos. Sólo 2 casos no recibieron tratamiento previo al ingreso en nuestra Unidad (Gráfico 2A).

\section{Tratamiento específico para la NET en la UQ-HGUA}

Se decidió tratar con corticoides sistémicos (Gráfico 2B) a 7 pacientes $(43,75 \%)$. El otro $37,5 \%$ recibió exclusivamente tratamiento de soporte (fluidoterapia, antibioterapia en algunos casos y soporte nutricional) y el $25 \%$ tratamiento con inmunoglobulinas intravenosas (IG iv) a dosis de $1 \mathrm{mg} / \mathrm{kg}$ /día durante 3 días (durante su administración, no hubo ningún efecto adverso en los pacientes tratados).

\section{Número de días de ingreso en la UQ-HGUA}

El número medio de días fue de 18,16. Destacamos a los pacientes 15 y 16 que solo estuvieron ingresados 1 día (ambos fallecieron de manera fulminante) y a los pacien-

Gráfico 2. Porcentaje de pacientes clasificados en relación al tipo de tratamiento administrado: A. previo al ingreso y B. una vez ingresados en la UQ-HGUA.

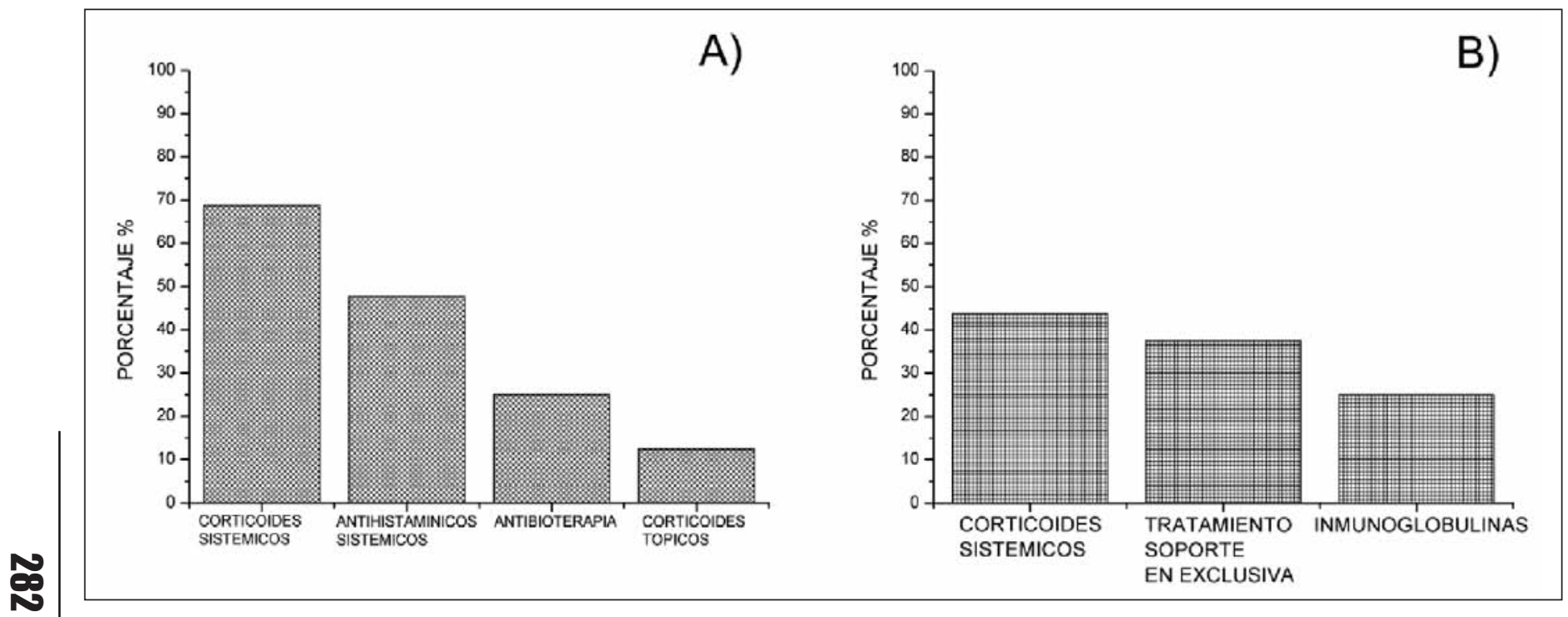


Tabla II. Descripción de los episodios de NET

\begin{tabular}{|c|c|c|c|c|c|c|c|}
\hline $\mathbf{N}^{\circ}$ & $\begin{array}{l}\text { Atención } \\
\text { previa } \\
\text { al ingreso }\end{array}$ & $\begin{array}{c}\text { Tto. } \\
\text { previo al ingreso }\end{array}$ & $\begin{array}{c}\text { Tto. } \\
\text { en UQ-HGUA }\end{array}$ & Secuelas & SCD & Diagnóstico & SCORTEN \\
\hline 1 & Pediatría & Corticoides iv & Corticoides iv & $\begin{array}{l}\text { Queratitis y úlceras } \\
\text { corneales que precisaron } \\
\text { trasplantes corneales }\end{array}$ & 50 & NET & 2 \\
\hline 2 & Hospital nivel II & $\begin{array}{l}\text { Corticoides iv, } \\
\text { Antihistamínico iv }\end{array}$ & Tto. de soporte & No secuelas & 50 & NET & 3 \\
\hline 3 & Hospital nivel II & $\begin{array}{l}\text { Corticoides iv, } \\
\text { Antihistamínico iv }\end{array}$ & Tto. de soporte & Éxitus & 17 & NET-SSJ & 4 \\
\hline 4 & Hospital nivel II & $\begin{array}{l}\text { Corticoides iv, } \\
\text { Antihistamínico iv }\end{array}$ & Tto. de soporte & Úlceras corneales & 60 & NET & 2 \\
\hline 5 & $\begin{array}{l}\text { Atención primaria y } \\
\text { Hospital nivel II }\end{array}$ & $\begin{array}{l}\text { Corticoides iv, } \\
\text { Antihistamínico iv }\end{array}$ & Corticoides vo & No secuelas & 36 & NET & 1 \\
\hline 6 & Hospital privado & $\begin{array}{l}\text { Corticoides iv, } \\
\text { Pentoxifilina, } \\
\text { Antibioterapia }\end{array}$ & Corticoides iv & Éxitus & 50 & NET & 2 \\
\hline 7 & $\begin{array}{l}\text { Atención primaria y } \\
\text { Hospital nivel II }\end{array}$ & $\begin{array}{l}\text { Corticoides vo, } \\
\text { Antihistamínico vo }\end{array}$ & IG iv & No secuelas & 70 & NET & 2 \\
\hline 8 & Hospital nivel II & $\begin{array}{l}\text { Corticoides iv, } \\
\text { Antibioterapia }\end{array}$ & Tto. de soporte & $\begin{array}{l}\text { Hemiparesia por recidiva } \\
\text { tumoral de astrocitoma }\end{array}$ & 15 & NET-SSJ & 4 \\
\hline 9 & Hospital nivel II & No tto. previo & Corticoides iv & No secuelas & 67 & NET & 3 \\
\hline 10 & $\begin{array}{l}\text { Atención primaria y } \\
\text { Hospital nivel II }\end{array}$ & Corticoides tópicos & $\begin{array}{l}\text { Corticoides iv, } \\
\text { IG iv }\end{array}$ & $\begin{array}{l}\text { Neumonía necrotizante } \\
\text { por Torulopsis Glabrata }\end{array}$ & 75 & NET & 3 \\
\hline 11 & Hospital nivel II & Corticoides iv & Tto. de soporte & No secuelas & 60 & NET & 2 \\
\hline 12 & Hospital nivel II & $\begin{array}{l}\text { Corticoides iv, } \\
\text { Antihistamínico iv, } \\
\text { Antibioterapia }\end{array}$ & Tto. de soporte & Sinequias conjuntivales & 40 & NET & 3 \\
\hline 13 & Hospital privado & No tto. previo & IG iv & No secuelas & 40 & NET & 2 \\
\hline 14 & Atención primaria & $\begin{array}{l}\text { Antihistamínico vo } \\
\text { Corticoide tópico }\end{array}$ & IG iv & No secuelas & 40 & NET & 3 \\
\hline 15 & Atención primaria & Corticoides vo & Corticoides iv & Éxitus & 90 & NET & 5 \\
\hline 16 & Hospital nivel II & Antibioterapia & Corticoides iv & Éxitus & 50 & NET & 5 \\
\hline
\end{tabular}

SCD: Superficie corporal desepidermizada; IG: inmunoglobulina; iv: intravenoso; vo: vía oral; NET: Necrolisis Epidérimica Tóxica; Tto: tratamiento.

tes 1,4 y 11 que estuvieron ingresados 1 mes o más, sobre todo el 1 que estuvo 45 debido a graves secuelas oculares.

\section{Mortalidad}

Durante el ingreso hospitalario fallecieron 4 pacientes (25\%) de los cuales el paciente 3 fue diagnosticado como SSJ-NET. La media de edad entre los pacientes fallecidos fue de 66,75 años, mayor que la de los pacientes no fallecidos que fue de 48,67 años.

\section{Secuelas}

El 56,25\% de los pacientes sufrió algún tipo de secuela tras el episodio de NET. La más frecuente entre los que superaron el cuadro fue la afectación ocular (3 pacientes, $18,75 \%$ ), con úlceras corneales y sinequias conjuntivales. Concretamente, el paciente 1 presentó graves perforaciones corneales que precisaron queratoplastias, trasplantes corneales y de limbo. 


\section{Superficie corporal desepidermizada y diagnóstico clínico-anatomopatológico}

La media de superficie corporal desepidermizada (SCD) fue del 50,63\%. El 87,5\% de los pacientes fue diagnosticado de NET tanto por resultados de anatomía patológica, como por una SCD mayor del $30 \%$. El 12,5\% fue diagnosticado de SSJ-NET por presentar una SCD de entre el 10 y el 30\%. La anatomía patológica de las biopsias obtenidas en estos pacientes mostró una marcada degeneración hidrópica de la membrana basal con vesiculación subepidérmica y necrosis de la epidermis supravesicular; presencia de edema dérmico papilar con infiltrado linfocítico, algunos eosinófilos y numerosos queratinocitos apoptóticos en la periferia de las ampollas.

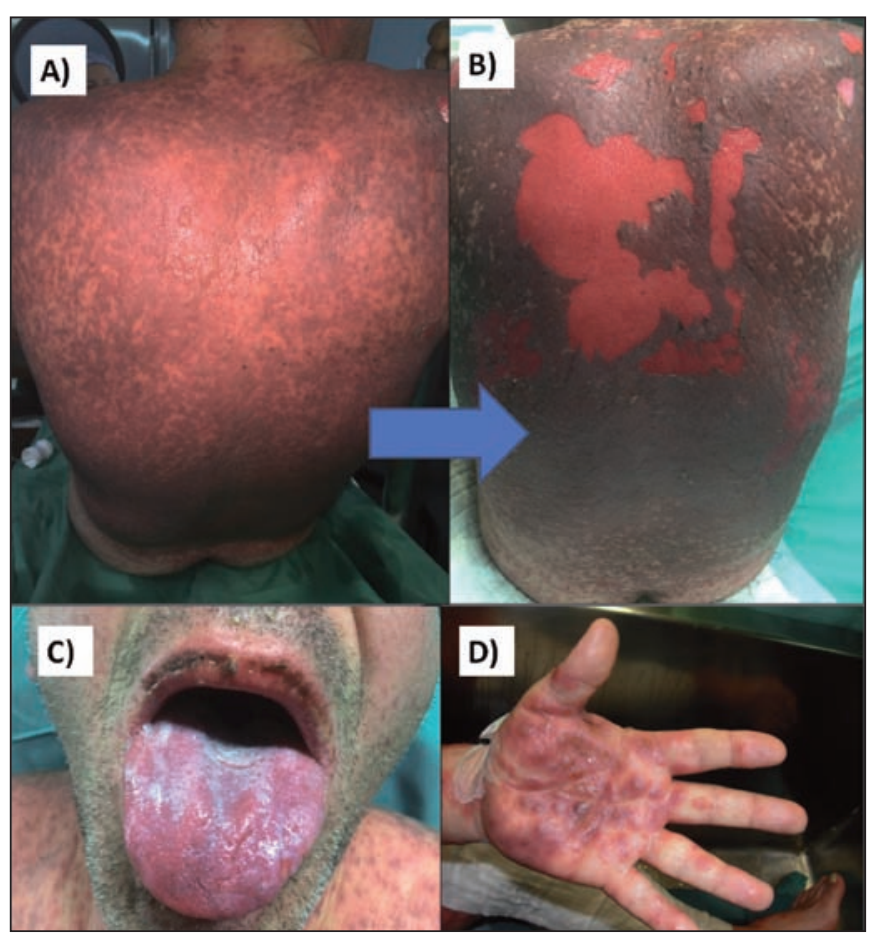

Fig. 1. A. Lesiones eritematosas al ingreso en espalda de paciente con NET, donde ya se pueden apreciar las primeras ampollas flácidas en el centro de la imagen B. Evolución de las lesiones a los 2 días de ingreso hospitalario, con despegamiento epidérmico. C. Afectación de la mucosa oral al ingreso. D. Lesiones eritematosas en palmas de manos al ingreso.

\section{Cuadro clínico y tipos de nutrición utilizados}

Todos los pacientes tuvieron una evolución clínica cronológica similar al inicio del cuadro:

- Cuadro de aparición brusca, tras un pródromo pseudocatarral.

- Empeoramiento del estado general con la aparición de lesiones eritematosas dolorosas, inicialmente distribuidas de forma simétrica en cara y tronco, con tendencia a la extensión hacia partes acras (Fig. 1). Estos síntomas solían preceder a las lesiones cutáneas de 1 a 3 días según los registros. El cuadro se acompañó de fiebre.

- Las lesiones evolucionaron desde máculo-pápulas eritematosas hacia un desprendimiento de la epidermis, con aparición de necrosis y ampollas flácidas (signo de Nikolsky positivo).

- Culminación del cuadro con el despegamiento de amplias zonas epidérmicas que dejaban erosiones exudativas (Fig. 1B).

El 100\% de los pacientes debutó con fiebre (entre 38 y $40{ }^{\circ} \mathrm{C}$ ); el $87,5 \%$ con afectación de las mucosas (oral, genital y oral), y el 18,75\% sufrió afectación pulmonar (Gráfico 3A). De estos últimos (3 en total), 2 presentaron hemoptisis al inicio del cuadro. Según la intensidad de afectación de la mucosa oral los pacientes recibieron distintos tipos de nutrición, para lo cual se empleó la vía oral en el 37,5\%, nutrición enteral por sonda nasogástrica en el $31,25 \%$, nutrición parenteral en un $25 \%$ y combinación de nutrición parenteral y enteral por sonda nasogástrica en el 6,25\% (Gráfico 3B).

\section{Balance de fluidos}

En todos los pacientes revisados se realizó un control exhaustivo del balance de entrada y salida de fluidos con colocación de sonda vesical y control de diuresis horaria durante las 2 primeras semanas de ingreso. Se utilizó al ingreso fluidoterapia de soporte con cristaloides, con volúmenes entre 4 y $2 \mathrm{ml} / \mathrm{kg} / \% \mathrm{SCD}$ según la diuresis y la tensión arterial de cada paciente. La mayoría presentó un

Gráfico 3. A. Porcentaje de pacientes con fiebre, afectación de mucosas (oral, genital y ocular) y afectación pulmonar. B. Clasificación de los pacientes según el tipo de nutrición administrada durante la primera semana de ingreso.

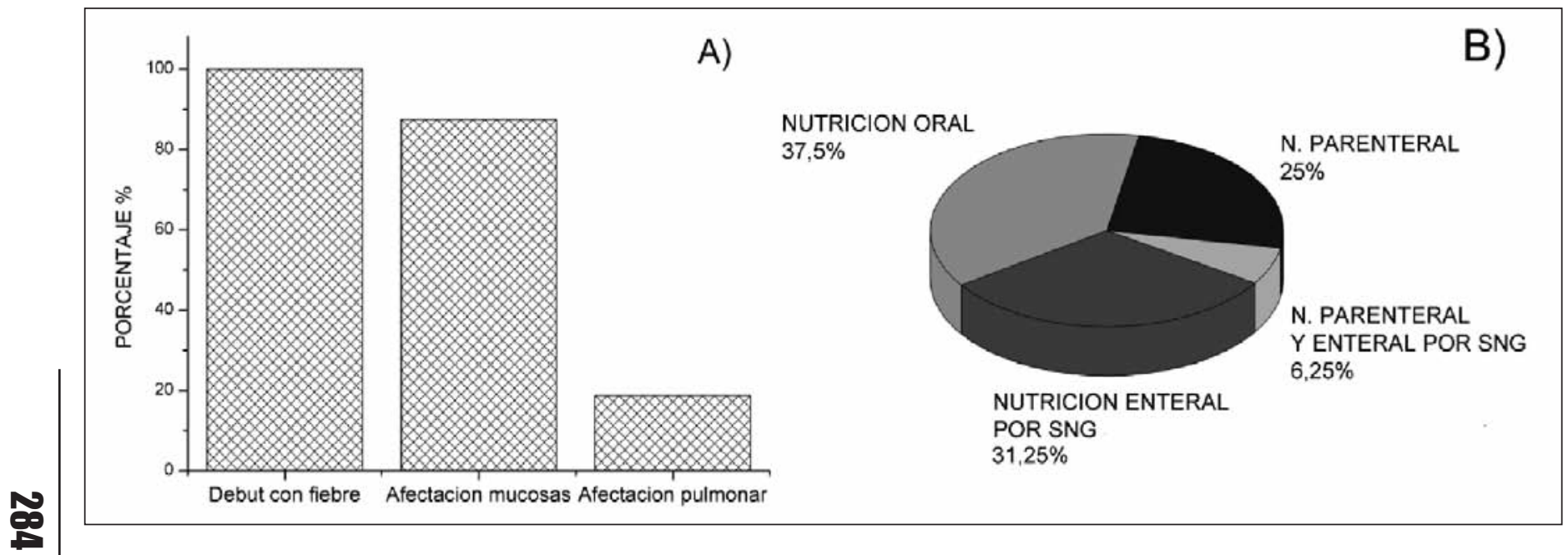




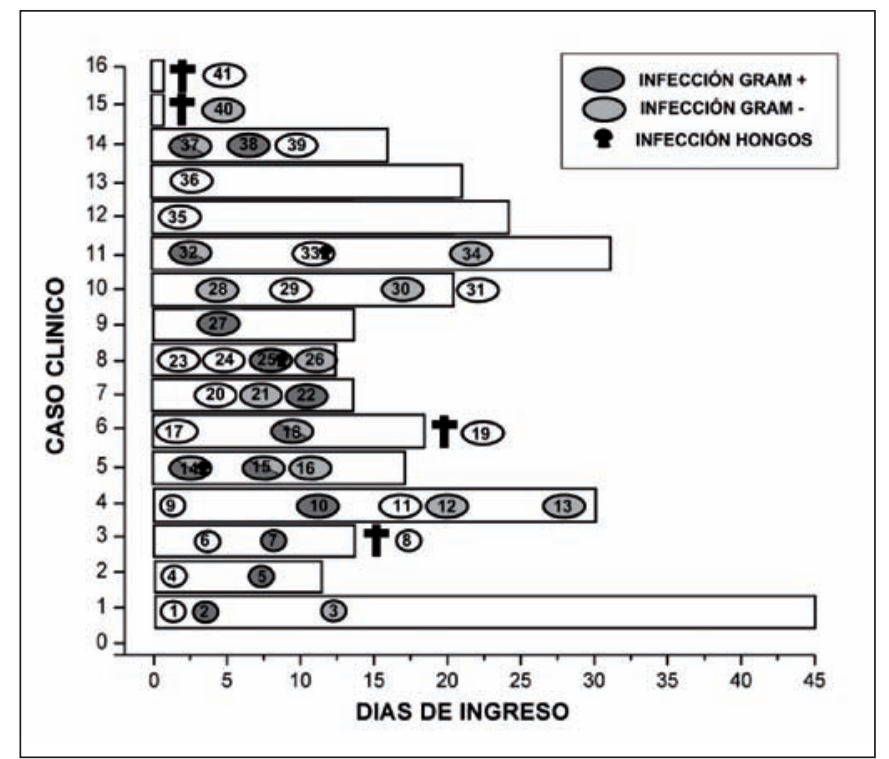

Fig. 2. Esquema temporal de eventos infecciosos durante los días que los pacientes estuvieron ingresados en la UQ-HGUA. La numeración dentro de las barras corresponde a los distintos eventos infecciosos y/o el comienzo de un tratamiento antibiótico asociado, ordenados temporalmente en relación con la duración de su estancia (la información correspondiente a cada evento infeccioso se encuentra detallada en la Tabla III). t= Éxitus.

balance adecuado de líquidos con esta perfusión, excepto el paciente 3 que sufrió un episodio de insuficiencia cardiaca congestiva complicada que provocó su fallecimiento.

\section{Evolución de la patología durante el ingreso y trata- miento antibiótico asociado}

En la Fig. 2 y la Tabla III resumimos la evolución temporal de los eventos infecciosos durante los días que los pacientes estuvieron ingresados en la UQ-HGUA. Como se puede apreciar en la distribución de la Fig. 2, la mayoría de las infecciones por Gram+ ocurrieron en los 10 primeros días de ingreso, pasando a tomar protagonismo las infecciones por Gram-a partir del día 7. Destacamos que el $43,75 \%$ de los pacientes recibió profilaxis antibiótica al ingreso en la UQ-HGUA; a pesar de ello la sepsis fue la complicación más habitual durante el ingreso, afectando a un 56,25\% del total de los pacientes. El género Acinetobacter apareció en los cultivos de punta de catéter venoso central de 4 pacientes ( $25 \%$ del total), en los hemocultivos de 2 pacientes y en 1 paciente en el cultivo de las heridas por despidermización. También se encontró Pseudomonas aeruginosa en los hemocultivos de 2 pacientes, en 1 en el cultivo de punta de catéter venoso central y en otro en el cultivo de las heridas por despidermización. Otros patógenos agresivos encontrados en hemocultivos o cultivo de punta de catéter fueron Gram+ tipo SARM, Staphylococcus epidermidis, Staphylococcus hominis hominis, y los Gram- Escherichia coli, Enterococcus faecalis, Enterobacter cloacae y Klebsiella pneumoniae productora de BLEE (ß-lactamasas de espectro extendido).
Sólo hubo 3 casos de cultivo positivo a levaduras, de los cuales 2 fueron en cultivos de lesiones en piel y 1 en hemocultivo. Todas las levaduras encontradas fueron del género Cándida. En cuanto a los cultivos de las heridas por despidermización, destacamos la presencia de los géneros Staphylococcus, Enterococcus y Enterobacter.

Clasificamos a los pacientes del estudio en 4 grupos (Gráfico 4) según el tratamiento recibido: 11 en el grupo de pacientes tratados con corticoides sistémicos previos al ingreso; 7 en el grupo de pacientes tratados con corticoides sistémicos al ingreso en la UQ-HGUA; 4 en el grupo de pacientes tratados con IG iv al ingreso; y 6 en el grupo de pacientes tratados con soporte al ingreso. En esta clasificación apreciamos que existe un porcentaje similar de cultivos positivos en hemocultivos y/o punta de catéter de vía central para los grupos de tratamiento con corticoides sistémicos, tanto previo como al ingreso, y para el grupo tratado con IG iv $(63,64 \%, 71,43 \%$ y $75 \%$, respectivamente). Sin embargo, para el grupo de pacientes en los que se aplicó tratamiento de soporte, encontramos un porcentaje de cultivos positivos menor $(33,33 \%)$ a pesar de coincidir el que todos ellos recibieron corticoides sistémicos previos al ingreso en la UQ-HGUA.

\section{SCORTEN en las primeras 24 horas de ingreso}

Calculamos el SCORTEN para los 16 pacientes (promedio SCORTEN 2,87, rango 1-5) en las primeras 24 horas de ingreso en la UQ-HGUA (Tabla IV). La suma de las probabilidades predichas de morir por NET fue de $32 \%$ o 5 muertes, cifra superior a los datos reales (mortalidad real $25 \%$ ).

De los 4 pacientes fallecidos, 3 habían recibido corticoides intravenosos según peso (1-1,5 $\mathrm{mg} / \mathrm{kg} / \mathrm{día})$ y el otro tratamiento de soporte. En el grupo de pacientes tratados con IG iv el SCORTEN predijo 1 defunción, mientras que la mortalidad real fue de 0 pacientes. En el grupo de pacientes tratados con corticoides la mortalidad predicha fue de 2,3 muertes y la real de 3 defunciones. En el grupo de pacientes tratados con medidas de soporte calculamos una mortalidad predicha de 2 y la real fue de 1 defunción.

\section{Tratamiento de las lesiones por despegamiento}

El 62,5\% de los pacientes recibió curas con una mezcla tópica de antibiótico y desbridante, que en la mayoría de los casos consistió en mupirocina y clostridiopeptidasa sobre un suporte de tul graso. El 25\% fue tratado con desbridamiento quirúrgico y colocación en quirófano de láminas de Biobrane ${ }^{\circledR}$ (sustituto de piel biosintético) sobre las zonas desepidermizadas. El 12,5\% de los pacientes (a partir de 2007) fue tratado con apósitos de plata nanocristalina (Acticoat Flex ${ }^{\circledR}$ ).

Durante el seguimiento de las curas de las zonas desepidermizadas no apreciamos diferencias significativas a favor de alguno de los tratamientos mencionados, siendo más barato el tratamiento tópico con antibiótico y desbridante. Los pacientes tratados con desbridamiento 
Tabla III. Evolución temporal de los eventos infecciosos durante los días de ingreso en la UQ-HGUA.

\begin{tabular}{|c|c|c|c|}
\hline $\mathbf{N}^{\mathbf{o}}$ & Evento & Tratamiento antibiótico y causa del éxitus & Cultivos positivos \\
\hline \multirow{3}{*}{1} & 1) & $1^{\circ}$ día profilaxis antibiótica con eritromicina & \\
\hline & 2) & $3^{\circ}$ día cambio a vancomicina y aztreonam & $\begin{array}{l}\text { Staphylococcus aureus resistente a meticilina (SARM) } \\
\text { en hemocultivos }\end{array}$ \\
\hline & 3) & $13^{\circ}$ día cambio a imipenem y amikacina & Acinetobacter anitratus en hemocultivos \\
\hline \multirow[t]{2}{*}{2} & 4) & $\begin{array}{l}1^{\circ} \text { día profilaxis antibiótica con vancomincina y } \\
\text { tobramicina }\end{array}$ & \\
\hline & 5) & & $7^{\circ}$ día Staphylococcus epidermidis en lesiones piel \\
\hline \multirow[t]{3}{*}{3} & 6) & $4^{\text {o }}$ día cloxacilina & \\
\hline & 7) & $8^{\circ}$ día cambio a piperacilina + tazobactam & $\begin{array}{l}\text { Staphylococcus aureus sensible a meticilina (SASM) en } \\
\text { lesiones piel }\end{array}$ \\
\hline & 8) & $\begin{array}{l}14^{\circ} \text { día éxitus por insuficiencia cardiaca } \\
\text { congestiva complicada }\end{array}$ & \\
\hline \multirow[t]{5}{*}{4} & 9) & $\begin{array}{l}1^{\circ} \text { día profilaxis antibiótica con cloxacilina } \\
\text { y gentamicina }\end{array}$ & \\
\hline & 10) & $12^{\circ}$ día cambio a vancomicina y ceftriaxona & $\begin{array}{l}\text { Staphylococcus epidermidis y Enterococcus faecalis en } \\
\text { lesiones piel }\end{array}$ \\
\hline & 11) & $18^{\circ}$ día cambio a vancomicina y cefepima & \\
\hline & 12) & $20^{\circ}$ día cambio a gentamicina y ceftriaxona & Escherichia coli en hemocultivos \\
\hline & 13) & & $\begin{array}{l}28^{\circ} \text { día Acinetobacter baumannii en catéter venoso cen- } \\
\text { tral retirado por alta hospitalaria }\end{array}$ \\
\hline \multirow[t]{3}{*}{5} & 14) & & $\begin{array}{l}3^{\circ} \text { día Staphylococcus epidermidis, Staphylococcus } \\
\text { auricularis, Enterobacter cloacae, Acinetobacter } \\
\text { baumannii, Enterococcus faecalis, y Candida albicans } \\
\text { en lesiones piel }\end{array}$ \\
\hline & 15) & & $\begin{array}{l}8^{\circ} \text { día Staphylococcus epidermidis, Acinetobacter } \\
\text { baumannii, Enterococcus faecalis, Enterobacter } \\
\text { cloacae, Klebsiella pneumoniae y Streptococcusmitis } \\
\text { en lesiones piel }\end{array}$ \\
\hline & 16) & $9^{\circ}$ día imipenem y amikacina & $\begin{array}{l}\text { Enterobacter cloacae y Acinetobacter lwoffii en catéter } \\
\text { venoso central y Streptococcus mitis en lesiones piel }\end{array}$ \\
\hline \multirow[t]{3}{*}{6} & 17) & $1^{\circ}$ día profilaxis antibiótica con meropenem & \\
\hline & 18) & $9^{\circ}$ día cambio a vancomicina, ciprofloxacino y colistina & $\begin{array}{l}\text { Acinetobacter baumannii, Staphylococcus epidermidis } \\
\text { multirresistente y Enterococcus faecalis en hemocultivos }\end{array}$ \\
\hline & 19) & $18^{\circ}$ día éxitus por shock séptico & \\
\hline \multirow[t]{3}{*}{7} & 20) & $\begin{array}{l}4^{\circ} \text { día cloxacilina y ceftazidima por fiebre } \\
\text { mantenida desde ingreso }\end{array}$ & \\
\hline & 21) & $7^{\circ}$ día cambio a ceftazidima y meropenem & $\begin{array}{l}\text { Klebsiella Pneumoniae productora de BLEE (ß-lactama- } \\
\text { sas de espectro extendido) en hemocultivos }\end{array}$ \\
\hline & 22) & $9^{\circ}$ día se añade vancomicina & SARM en lesiones piel \\
\hline \multirow[t]{4}{*}{8} & 23) & $\begin{array}{l}1^{\circ} \text { día linezolid, ceftazidima y ciprofloxacino } \\
\text { iniciado previamente en anterior hospital }\end{array}$ & \\
\hline & 24) & $3^{\circ}$ día cambio a cloxacilina y gentamicina & \\
\hline & 25) & $5^{\circ}$ día cambio a vancomicina y ceftazidima & Staphylococcus epidermidis y Candida albicans en lesiones piel \\
\hline & 26) & $10^{\circ}$ día cambio a meropenem y amikacina & Acinetobacter baumannii en catéter venoso central \\
\hline
\end{tabular}


Tabla III. Evolución temporal de los eventos infecciosos durante los días de ingreso en la UQ-HGUA (continuación).

\begin{tabular}{|c|c|c|c|}
\hline $\mathbf{N}^{\mathbf{o}}$ & Evento & Tratamiento antibiótico y causa del éxitus & Cultivos positivos \\
\hline 9 & 27) & & $4^{\circ}$ día Enterococcus faecalis en lesiones piel \\
\hline \multirow[t]{4}{*}{10} & 28) & $4^{\circ}$ día inicio de meropenem & Pseudomonas aeruginosa en lesiones piel \\
\hline & 29) & $9^{\circ}$ día se añaden fluconazol y ciprofloxacino & \\
\hline & 30) & $17^{\circ}$ día cambio a imipenem y colistina & $\begin{array}{l}\text { Acinetobacter baumannii en en catéter venoso central y } \\
\text { en esputo }\end{array}$ \\
\hline & 31) & $\begin{array}{l}20^{\circ} \text { día continúa tratamiento antibiótico fuera de } \\
\text { UQ. por resolución cuadro NET }\end{array}$ & \\
\hline \multirow[t]{3}{*}{11} & 32) & $2^{\circ}$ día vancomicina y meropenem & $\begin{array}{l}\text { Escherichia coli productor de BLEE y Enterococcus } \\
\text { faecalis en lesiones piel y Escherichia coli en urocultivo }\end{array}$ \\
\hline & 33) & $12^{\circ}$ día se añade fluconazol & $\begin{array}{l}\text { Candida parapsilosis en hemocultivos y Candida } \\
\text { famata en lesiones piel }\end{array}$ \\
\hline & 34) & $\begin{array}{l}22^{\circ} \text { se cambio ciprofloxacino en lugar de } \\
\text { vancomicina }\end{array}$ & $\begin{array}{l}\text { Pseudomonas aeruginosa en catéter venoso central y } \\
\text { hemocultivo }\end{array}$ \\
\hline 12 & $35)$ & $\begin{array}{l}1^{\circ} \text { día profilaxis antibiótica con tobramicina } \\
\text { y ceftazidima }\end{array}$ & \\
\hline 13 & 36) & $\begin{array}{l}2^{\circ} \text { día profilaxis antibiótica con cloxacilina } \\
\text { y ceftriaxona }\end{array}$ & \\
\hline \multirow[t]{3}{*}{14} & $37)$ & $2^{o}$ día vancomicina y gentamicina & $\begin{array}{l}\text { Enterococcus faecalis, Staphylococcus epidermidis, } \\
\text { Staphylococcus haemolyticus, Klebsiella oxytoca y } \\
\text { Serratia marcescens en lesiones piel }\end{array}$ \\
\hline & 38) & $8^{\circ}$ día cambio a linezolid y gentamicina & Staphylococcus hominis hominis en hemocultivo \\
\hline & 39) & $9^{\circ}$ día cambio de meropenem & \\
\hline 15 & 40) & $\begin{array}{l}1^{\circ} \text { día con vancomicina por sospecha de cuadro } \\
\text { séptico. }\end{array}$ & $\begin{array}{l}\text { Hemocultivos postmortem positivos para Pseudomonas } \\
\text { aeruginosa. Éxitus por shock séptico }\end{array}$ \\
\hline 16 & 41) & $1^{\circ}$ día éxitus por fracaso multiorgánico & \\
\hline
\end{tabular}

quirúrgico y Biobrane ${ }^{\circledR}$ no requirieron un segundo desbridamiento quirúrgico, aunque alguno de ellos precisó retirada del sustituto dérmico en quirófano. No se utilizaron en ningún caso injertos de piel para cobertura. La elección de cada uno de los tratamientos dependió de la experiencia y opinión de cada facultativo. La reepitelización completa de la piel se produjo en 3 semanas siempre que se mantuvo un adecuado control microbiológico local y un adecuado control nutricional. Se realizaron controles microbiológicos mediante cultivo de las lesiones regularmente, con frecuencia semanal.

\section{Discusión}

A pesar de la rareza de la NET es necesario un profundo conocimiento de esta entidad nosológica para el facultativo responsable de una UQ, no sólo por la variedad de órganos y sistemas que afecta, sino también por la morbimortalidad que asocia. La incidencia registrada en nuestro hospital fue de 0,43 casos/millón/año (tomando como población media de la provincia de Alicante
1,62 millones de habitantes en los últimos 23 años). Este valor es algo menor en comparación con lo publicado en otros trabajos internacionales, pudiendo deberse a que parte de los pacientes con NET pueden no haber llegado a ingresar en la UQ-HGUA debido a su fallecimiento prematuro antes del traslado a nuestra Unidad, o a que han podido ser trasladados a la UQ del Hospital la Fe de Valencia, centro de referencia de grandes quemados para la Comunidad Valenciana en España. En comparación con otros estudios de nuestro entorno la incidencia de NET es similar si tenemos en cuenta el número de habitantes que abarca este hospital (4).

La distribución por sexos se asemeja a lo publicado, siendo el 68,75\% mujeres y el $31,25 \%$ hombres. La edad media fue de 53,19 años, similar a la que aparece en otros estudios (5). Debido a esta media de edad, es habitual encontrar en estos pacientes comorbilidades asociadas, como hipertensión arterial o diabetes, así como la toma de varios fármacos a la vez.

La media de SCD fue entre nuestros pacientes del $50,63 \%$, similar también a la de otros estudios previos $(4,5)$. 
Gráfico 4. Porcentaje de pacientes con cultivos positivos clasificados en $\mathbf{4}$ grupos según su tratamiento asociado: grupo de pacientes tratados con corticoides sistémicos previos al ingreso en la UQ-HGUA, grupo de pacientes tratados con corticoides sistémicos al ingreso en la UQ-HGUA, grupo de pacientes tratados con IG iv al ingreso en la UQ-HGUA y grupo de pacientes tratados con tratamiento de soporte al ingreso en la UQ-HGUA.

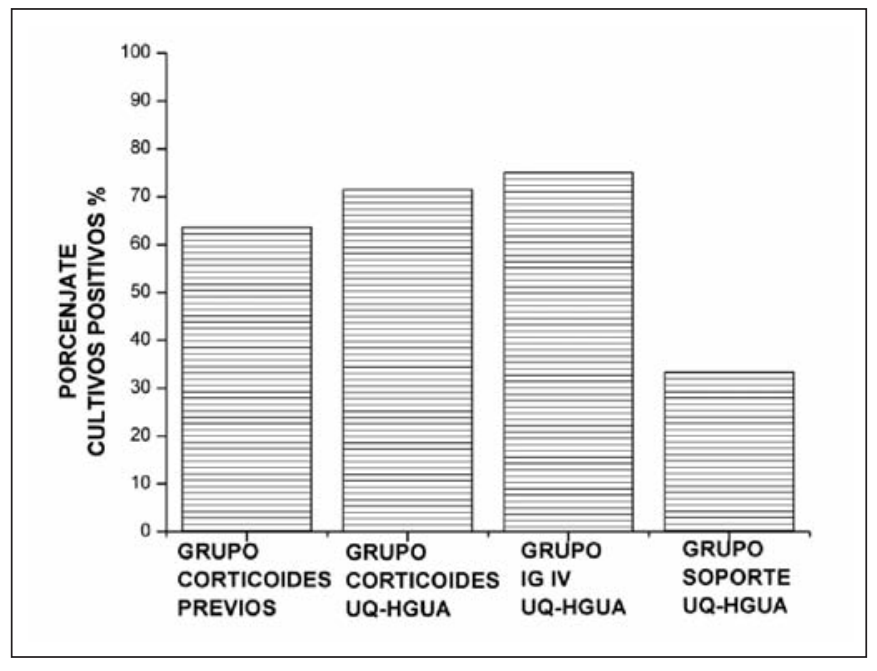

Tabla IV. SCORTEN de los pacientes

\begin{tabular}{|c|c|c|c|}
\hline \multicolumn{2}{|c|}{} & \multicolumn{2}{c|}{ Muertes, n (\%) } \\
\hline SCORTEN & Pacientes, n & Predicho & Real \\
\hline 1 & 1 & $0,04(3,9)$ & $0(0)$ \\
\hline 2 & 6 & $0,7(12,2)$ & $1(16,7)$ \\
\hline 3 & 5 & $1,6(32,4)$ & $0(0)$ \\
\hline 4 & 2 & $1,2(62,2)$ & $1(50)$ \\
\hline 5 & 2 & $1,7(85)$ & $2(100)$ \\
\hline Total & $\mathbf{1 6}$ & $\mathbf{5 , 2}(\mathbf{3 2}, \mathbf{5})$ & $\mathbf{4}(\mathbf{2 5})$ \\
\hline
\end{tabular}

La correlación entre la ingesta del fármaco y la aparición de la sintomatología compatible con el cuadro de NET no es inmediata, se desarrolla entre 1 y 8 semanas desde la administración del fármaco en sospecha, con una media de tiempo de inicio que va desde los 6 días a las 2 semanas (6,7). En nuestro estudio fue de 11,7 días con un intervalo de entre 1 y 60 días, similar a los valores obtenidos en nuestro entorno (4). No existen test que permitan ser concluyentes en la relación entre un caso y un fármaco en particular; además es frecuente en estos pacientes la ingesta de varios fármacos a la vez, como ocurrió en la mayoría de nuestros casos. Por ello muchas veces sólo podemos hablar de un fármaco desencadenante como sospechoso. Ha habido intentos en la bibliografía para desarrollar algoritmos de causalidad de fármaco, como el publicado por Sassolas et al. (ALDEN) (8), pero que todavía necesitan ser evaluados más pormenorizadamente. Existen datos de que tras la readministración de la droga implicada la NET puede desarrollarse en tan solo horas, por lo que en el futuro el pa- ciente tiene que tener muy en cuenta este dato. Hasta la fecha, ninguna prueba diagnóstica es infalible y la más fiable, la readministración del fármaco, es éticamente inaceptable por ser potencialmente letal.

La demora media entre el inicio de los síntomas y el ingreso en la UQ-HGUA fue en nuestro estudio de 6,18 días, ligeramente superior a la de los hospitales de nuestro entorno (4). El curso de estas enfermedades es muy similar al de los pacientes con quemaduras extensas, por lo que el transporte temprano del paciente a una Unidad de Quemados y el enfoque multidisciplinario a la hora de diagnosticar la patología es básico para llevar a cabo un tratamiento adecuado. McGee et al. (7) publican un estudio en el que muestran que con el transporte a una Unidad de Quemados dentro de los primeros 7 días, la mortalidad es significativamente menor, del $4 \%$, en comparación con la que se produce si el ingreso se realiza más allá del $7^{\circ}$ día que se eleva hasta el $83 \%$. Un error muy habitual es no pensar que la NET constituye una verdadera urgencia sistémica, lo que conduce a un reconocimiento tardío de la entidad y al traslado del paciente a la Unidad de Quemados cuando el proceso ya está muy evolucionado. En nuestro caso, la media de días entre la aparición de los síntomas y el ingreso en la UQ-HGUA, de 6,18 días, es mejor que la que presenta un estudio checo similar publicado en 2012, de 9,6 días (9), pero levemente superior a la de otros hospitales de nuestro entorno (4), en los que es de 4,4 días, por lo que pensamos que es necesario tomar conciencia de la importancia de reconocer este tipo de patología de forma temprana en los Servicios de Urgencias de los hospitales comarcales para evitar tanto la demora en el transporte a una UQ, como el inicio de tratamientos que no estén justificados en estos pacientes. De hecho, a la mayoría de los pacientes de nuestro estudio, $68,75 \%$, les habían administrado corticoides sistémicos como tratamiento principal antes del ingreso en la UQ-HGUA. Es un hecho conocido que el tratamiento con corticoides no se recomienda en muchos centros hospitalarios debido al posible aumento de la morbilidad, la mortalidad y la duración de la hospitalización en comparación con las de los pacientes en los que no se empelan como tratamiento (10).

En nuestros pacientes, la NET podría atribuirse en la mitad de los casos a un sólo fármaco, siendo los antibióticos la clase farmacológica más habitual. En el estudio de vigilancia farmacológica European Severe Cutaneous Adverse Reaction (EuroSCAR) (11) y en el trabajo de Roujeau et al. (6), se catalogan los siguientes fármacos como de alto riesgo: nevirapina, lamotrigina, carbamacepina, fenitoína, fenobarbital, cotrimoxazol y otras sulfonamidas, sulfasalacina, alopurinol, AINES, aminopenicilinas, cefalosporinas y quinolonas. Excepto en el paciente 9 de nuestro estudio, cuyos fármacos fueron otros no habituales, y los 2 pacientes con agente causal desconocido, el resto de los casos de NET que registramos se atribuyeron a fármacos incluidos en la lista ante- 
rior. El patrón de frecuencias obtenido en nuestro estudio es parecido al de otros trabajos publicados previamente (6), siendo en nuestro caso los antibióticos y los AINES los más habituales, a diferencia de lo publicado por otros trabajos de nuestros entorno (4). También en nuestro estudio observamos que los fármacos asociados a mayor mortalidad son los antimetabolitos, destacando el alopurinol, y los antibióticos (11).

El diagnóstico diferencial de la NET incluye: el eritema multiforme major, el síndrome de la piel escaldada estafilocócico, la dermatosis por inmunoglobulina A lineal inducida por fármacos, la enfermedad injerto contra huésped, la pustulosis exantemática generalizada aguda, la reacción farmacológica con eosinofilia y síntomas sistémicos, y más comúnmente, un exantema morbiliforme generalizado por fármacos. La biopsia de piel es útil para distinguir la NET del resto de diagnósticos alternativos mencionados.

El diagnóstico de NET se hace sobre la base tanto de hallazgos histológicos como clínicos. Suele existir un pródromo a las manifestaciones mucocutáneas en forma de tos, rinorrea, fiebre, anorexia y malestar general (12). Los principales hallazgos clínicos incluyen: eritema, máculas oscuras o violáceas y lesiones morbiliformes atípicas. Estas lesiones cutáneas evolucionan a una ampolla por despegamiento epidérmico y a la necrosis franca de la piel con una tonalidad grisácea. La NET se suele propagar simétricamente desde la cara y el tronco hacia las extremidades (12). En nuestros pacientes, el 100\% debutó con fiebre y el 87,5\% presentó afectación de mucosas ocular, genital y oral, datos que se asemejan a los descritos en la bibliografía consultada (13). Las erosiones de piel y mucosas son de tamaños y formas irregulares y dolorosas. El signo de Nikolsky (levantamiento o desprendimiento de la epidermis por una suave presión lateral) y el signo de Asboe-Hansen (extensión lateral de las ampollas con la presión), son muy habituales.

El diagnóstico clínico se puede completar con una analítica sanguínea en la que se puede encontrar eosinofilia, linfocitosis con linfocitos atípicos, leucopenia, anemia normocítica, velocidad de sedimentación globular aumentada, tiempo de protrombina y tromboplastina alargados, hipoproteinemia, aumento de transaminasas e hipercolesterolemia, y con la recogida de muestras microbiológicas al ingreso de la piel desepidermizada, de mucosa nasal y rectal.

El número medio de días de ingreso de nuestros pacientes en la UQ-HGUA fue de 18,16 días, similar a la de otros estudios europeos (5).

El tratamiento primario para la NET consiste en la eliminación del agente causal, el traslado del paciente a una Unidad de Quemados o de Cuidados Intensivos y la administración de una adecuada terapia de soporte. Es fundamental la suspensión inmediata de todos los tratamientos sospechosos, ya que éste será el factor pronóstico más importante, por lo que se impone que todos los médicos conozcan esta entidad. Creemos que las Unidades de Quemados, por estar habituadas a tratar pacientes con daño epidérmico extenso, son el lugar ideal para enfocar el tratamiento de estos pacientes que se basará en el mantenimiento y reconstitución de la función barrera de la piel mediante curas de la piel desepitelizada por personal entrenado, control analgésico adecuado, equilibrio de fluidos, higiene oral con antisépticos y/o antifúngicos (clorhexidina o nistatina), tratamiento del prurito con antihistamínicos, prevención del daño ocular con valoración y seguimiento oftalmológico, y en un seguimiento cuidadoso de la aparición y tratamiento de la infección.

En contraste con el paciente quemado habitual, en la NET solo se afecta la epidermis y la lesión microvascular es menor, por lo que los requerimientos de líquidos y de electrolitos son menores que para quemaduras de la misma superficie. Las perdidas insensibles son de 2 a 3 litros/día en adultos con un 50\% SCD, por lo que con la administración inicial de $2 \mathrm{ml} / \mathrm{kg} / \%$ SCD (de 5-7 litros/24 horas para un caso de este tipo) sería suficiente para garantizar una diuresis adecuada y una correcta tensión arterial (14). Se recomienda mantener una diuresis de entre 0,5 a $1,0 \mathrm{ml} / \mathrm{kg} / \mathrm{h}$, evitando la sobrecarga de volumen. En los pacientes de nuestro estudio, en ocasiones se utilizaron cifras de entre 2 y $4 \mathrm{ml} / \mathrm{kg} / \%$ SCD según la diuresis horaria del paciente, ya que en las 2 primeras semanas de ingreso es fundamental monitorizar este parámetro.

El rol de la respuesta inmune en la fisiopatología de la NET ha inspirado el uso de diferentes fármacos con acciones sobre el sistema inmune, como corticoides sistémicos, inmunosupresores, agentes anti-TNF $\alpha$, plasmaféresis e IG iv.; pero los datos al respecto siguen siendo contradictorios. Los corticosteroides provocan resultados dispares; se han asociado con un aumento de la infección, de la duración de la estancia hospitalaria y de la mortalidad (15), si bien algunos estudios más recientes, como los de Kardaun et al. (16), sugieren un posible papel beneficioso si se administran de forma temprana, en dosis altas, y por períodos cortos de tiempo, provocando una reducción del impacto negativo en cuanto a la infección y a la epitelización de las heridas con respecto a lo observado en otros estudios con corticoides. En el mayor estudio retrospectivo publicado hasta la fecha sobre el tratamiento de la NET (EuroSCAR) (10), con 281 pacientes de Alemania y Francia tratados con corticoides, IG iv. o tratamiento de soporte en exclusiva, se encontró una reducción de la mortalidad en los pacientes de Alemania pero no en los de Francia al comparar el grupo de tratamiento con corticoides y el grupo de tratamiento de soporte. En nuestro estudio, los corticoides sistémicos fueron el tratamiento farmacológico más utilizado $(43,75 \%)$ debido a la creencia extendida que se tenía hasta hace pocos años entre los facultativos de que estaban indicados en la NET. Además, de los 4 pacientes fallecidos, 3 recibieron corticoides sistémicos iv. según peso y el otro recibió tratamiento de soporte, por lo que, 
en nuestra opinión, no es recomendable el uso de corticoides sistémicos como tratamiento farmacológico para esta enfermedad.

En general, en la última década, el tratamiento de la NET se ha alejado de los corticoides hacia otro tipo de tratamientos como las IG iv., ya que regulan pasos cruciales en la respuesta inmunitaria mediada por células $\mathrm{T}$ además de contener anticuerpos anti-Fas (inhibición in vitro de la apoptosis de los queratinocitos mediada por Fas-FasL). Existen estudios que muestran una disminución de la mortalidad cuando se emplean IG iv., con porcentajes de supervivencia del $88 \%, 94 \%$ y $100 \%$ con dosis totales de 2,7, 4 y $3,4 \mathrm{~g} / \mathrm{kg}$ respectivamente (17). A pesar de estos resultados, otros estudios no encuentra beneficios en las tasas de mortalidad con dosis totales de IG iv. de entre 1,6 o 2,8 g/ $/ \mathrm{kg}$ en comparación con los tratamientos de soporte (18). Tampoco en el estudio EuroSCAR se encontró una mejoría de la mortalidad en comparación con el grupo de tratamiento de soporte (10). Solo existe un metaanálisis sobre la eficacia de la IG iv. (19) en el que los pacientes tratados con dosis altas, mayores de $2 \mathrm{~g} / \mathrm{kg}$, presentan una mortalidad significativamente menor que los tratados con dosis bajas: del 18,9\% frente al 50\% respectivamente; sin embargo, el modelo de regresión logística multivariante indicó que la dosis de IG iv. no influía en la mortalidad.

Están apareciendo nuevos estudios que emplean una combinación de corticoides e IG iv. con resultados positivos (20), pero aún se necesitan más investigaciones para valorar su eficacia.

En nuestro grupo de estudio ningún paciente tratado con IG iv. falleció, aunque la media de SCORTEN fue 2,5 menor en el caso de los pacientes tratados con corticoides sistémicos o con tratamiento de soporte cuyo valor fue 3 . Sin embargo, el porcentaje de cultivos positivos que obtuvimos en este grupo de pacientes fue comparable al del grupo de pacientes tratados con corticoides sistémicos y casi el doble que en el caso del grupo con tratamiento soporte.

La plasmaféresis es otra de las terapias que se ha utilizado en pacientes con NET agresiva refractaria a la terapia de soporte y/o a los corticoides (21), obteniendo una mejoría rápida. Sin embargo, es un tratamiento caro y no existe acuerdo en cuanto a su eficacia en NET. Otras terapias adyuvantes para el tratamiento de la NET sobre las que hay escasos datos que apoyen su uso son: inhibidores de TNF- $\alpha$, factor estimulante de colonias de granulocitos, $\mathrm{N}$-acetilcisteína, y ciclosporina. Ejemplos de inhibidores de TNF- $\alpha$ son infliximab (22), etanercept y pentoxifilina. El factor estimulante de colonias de granulocitos parece tener resultados positivos en pacientes con NET y neutropenia. El interés por la ciclosporina se ha incrementado con el descubrimiento de la importancia de la granulisina, una proteína antimicrobiana presente en los linfocitos T en la apoptosis de la NET. Hay varios estudios recientes que apoyan su posible papel en el tratamiento de esta enfermedad (23), con buena tolerancia en la mayoría de los pacientes y sin aumento de la tasa de infecciones. Sin embargo, también son necesarios por ahora más estudios para validar su eficacia.

En nuestro estudio la limitación más importante es el pequeño número de casos recogidos, por lo que no tenemos datos de peso para apoyar o rechazar ninguno de los tratamientos farmacológicos utilizados para la NET. Hemos visto una mayor mortalidad en el grupo de pacientes tratados con corticoides y un mayor número de cultivos positivos en el grupo tratado con corticoides y/o IG iv. Lo que sí parece claro es que todavía no existe suficiente evidencia del beneficio de ningún tratamiento específico de los que hemos comentado, lo que se debe a la falta de ensayos aleatorizados, controlados y comparativos que evalúen los resultados de los diferentes tratamientos sistémicos en términos de mortalidad, morbilidad o mejoría clínica de los pacientes.

Hasta donde sabemos, existen muy pocos estudios que analicen el componente infeccioso de los pacientes con NET, cuya importancia es crucial para su evolución, tal y como extrapolamos del hecho de que el 56,25\% de nuestros pacientes sufriera algún tipo de proceso séptico, complicación que fue la más habitual durante el ingreso. Como ocurre en el paciente quemado, las zonas desepidermizadas son inicialmente colonizadas sobre todo por organismos Gram+ que rápidamente, en torno a 1 semana, se ven reemplazados por Gram- con buena susceptibilidad antibiótica. Posteriormente aparecen otros organismos como bacterias Gram- más virulentas y con mayor resistencia antibiótica tipo Enterococo, Escherichia coli o Pseudomonas aeruginosa, Gram+ tipo SARM, u hongos tipo Candida, Aspergillus o Mucor. Así pues, la sepsis fue la complicación más habitual durante el ingreso por NET de los pacientes de nuestro grupo, tal y como describen también otros estudios (24). Destacan habitualmente los géneros S.Aureus y Pseudomonas spp, aunque en nuestro caso el que apareció en más ocasiones y con mayor virulencia fue el género Acinetobacter. De las 4 muertes registradas en nuestro seguimiento, 2 (50\%) fueron por shock séptico atribuido a patógenos como Acinetobacter baumannii, Staphylococcus epidermidis multirresistente, Enterococcus faecalis y Pseudomonas aeruginosa. Los catéteres venoso centrales (CVC) son fundamentales en el manejo de estos pacientes, ya que precisan la administración de fluidos, fármacos y en muchos casos, nutrición parenteral por la imposibilidad de utilizar la vía oral. Además, muchas veces los CVC se encuentran cerca de zonas desepidermizadas que pueden estar colonizadas por los patógenos que hemos comentado, por lo que se aumenta el riesgo de contaminación de la vía y la aparición de bacteriemias (25). De hecho, del total de los pacientes de nuestro estudio, el 43,75\% dió positivo para algún patógeno en el cultivo de punta de CVC, mientras que el $37,5 \%$ dio positivo en hemocultivos. De especial importancia fue la presencia en nuestros pacientes de los géneros Acinetobacter y Pseudomonas por su destacada mortalidad en el pa- 
ciente quemado. La impregnación de los CVC con antisépticos tipo clorhexidina es un método que reduce la proporción de infecciones en CVC, como aparece publicado en un reciente metaanálisis (26).

No está recomendado en esta patología el uso de antibióticos profilácticos a pesar de que en muchas ocasiones se compruebe la presencia de fiebre al ingreso $(12,27)$. Sin embargo, 7 pacientes $(43,75 \%)$ de nuestro grupo recibieron tratamiento profiláctico al ingreso en la UQ-HGUA. De ellos, más de la mitad sufrieron bacteriemias durante los días posteriores por patógenos resistentes a los antibióticos profilácticos utilizados, como es el caso del género Acinetobacter. De entre los pacientes fallecidos, 1 recibió profilaxis antibiótica con meropenem, a pesar de lo cual falleció por shock provocado por patógenos multirresistentes. Los antibióticos sistémicos sólo deberían administrarse en caso de sobreinfección demostrada o altamente probable, siendo recomendable el realizar frotis cutáneos sistemáticos cada 2-3 días para identificar cuanto antes los gérmenes implicados.

El tratamiento de las zonas afectadas es también crucial en el cuidado de los pacientes con NET. La epidermis se exfolia de la dermis justo en su unión, estando la capa basal de este despegamiento bien perfundida con restos de los queratinocitos basales en los anexos de la piel. No recomendamos el uso de sulfadiazina de plata tópica debido a los posibles efectos de sensibilización secundarios de las sulfonamidas, aunque los datos aportados por otros hospitales de nuestro entorno hablan de un uso seguro de la misma para la cura tópica de las zonas desepidermizadas por NET (4). Boorboor et al. (28) publican un estudio en el que comparan la cura con tratamiento antiséptico conservador y la cobertura con Biobrane ${ }^{\circledR}$, obteniendo reducción del dolor, movilización más temprana del paciente, reducción del tiempo de repitelización y reducción de los marcadores infecciosos en sangre en los pacientes tratados con Biobrane ${ }^{\circledR}$. El 25\% de nuestros pacientes fue tratado con desbridamiento quirúrgico y colocación de láminas de Biobrane ${ }^{\circledR}$, sin que hayamos podido apreciar una diferencia significativa en el tiempo de reepitelización en comparación con el uso de apósitos de plata nanocristalina o con curas diarias con mupirocina y clostridiopeptidasa sobre un suporte de tul graso. Sin embargo, en pacientes con superficies desepidermizadas grandes, por encima del 50-60\%, recomendamos las láminas de Biobrane ${ }^{\circledR} \mathrm{o}$ los apósitos de plata nanocristalina ya que no precisan retirada y cambio diario, lo que disminuye el dolor durante el proceso de cicatrización.

Debido a que la afectación de las mucosas es común en esta patología, los pacientes experimentan un intenso dolor durante la deglución que disminuye su ingesta oral. De hecho, el 25\% de los pacientes con afectación de mucosas de nuestro estudio precisó nutrición parenteral y el $6,25 \%$ la combinación de nutrición parenteral y enteral por sonda nasogástrica. Esto conlleva un aumento del riesgo de sepsis por la necesidad de utilizar CVC para la nutrición parenteral en un porcentaje alto de nuestros pacientes. Para compensar el estado catabólico y teniendo en cuenta la habitual disfagia de estos pacientes, se recomienda iniciar cuanto antes una nutrición enteral hipercalórica e hiperprotéica mediante sonda de silicona. Los requerimientos nutricionales pueden ser más bajos que en los pacientes quemados, tal y como aparece en un estudio que sugiere que los pacientes pediátricos con NET requieren un $22 \%$ menos de calorías/día en comparación con los pacientes quemados.

Los pacientes que sobreviven a la fase aguda de la NET tienen riesgo de sufrir secuelas. De hecho, el $56,25 \%$ de nuestros pacientes sufrieron algún tipo de secuela entre las que la no mortal más frecuente fue la afectación ocular. Las lesiones oculares son la complicación más común descrita en la literatura al respecto, con un $20 \%$ y un $79 \%$ de afectación de los pacientes $(29,30)$, e incluyen: síndrome de ojo seco, fotofobia, sensación de arena en los ojos, simbléfaron, cicatrización corneal, neovascularización de la córnea, xerosis corneal, agudeza visual reducida, ceguera y fibrosis subconjuntival. El síndrome del ojo seco es la complicación ocular más común e incluso puede ocurrir en pacientes que no han presentado afectación ocular aguda. La frecuencia de estas complicaciones requiere llevar a cabo una consulta oftalmológica temprana en todos los casos. La lesiones oculares en la NET pueden prevenirse mediante la aplicación de lubricación continua en el ojo, el uso de tratamiento antibiótico tópico y la lisis de las adherencias. En ocasiones son también necesarios otros procedimientos más invasivos, como el trasplante de membrana amniótica, reservado para aquellos pacientes con grandes áreas de desprendimiento epitelial que incluyen córnea, conjuntiva y párpado.

Por la cicatrización de otras mucosas puede aparecer además estenosis traqueal, esofágica y/o en la región anogenital. Son habituales también la despigmentación cutánea y las complicaciones dentales y sin embargo, son raras las complicaciones pulmonares como la bronquitis crónica y las bronquiectasias. No hay que minusvalorar tampoco las secuelas psicológicas y el temor de los pacientes ante cualquier tratamiento futuro con fármacos.

Nuestro estudio apoya la capacidad de SCORTEN para predecir la mortalidad en pacientes con NET en un hospital de tercer nivel como es el HGUA. En cada puntuación hubo una buena correlación entre muertes reales y pronosticadas, excepto para el valor SCORTEN 3 donde no hubo ninguna muerte registrada. SCORTEN debe realizarse en el día 1 y en el día 3 tras la admisión para optimizar su valor predictivo.

Para finalizar nuestro estudio, y una vez realizado todo el análisis expuesto, proponemos un protocolo de actuación ante los pacientes con NET o SSJ-NET que requieran ingreso en una UQ (Fig. 3) basado en los siguientes aspectos fundamentales: 
SUSPENDER corticoides si estaban pautados, medicamentos innecesarios y/o medicamentos sospechosos. -NO UTILIZAR PROFILAXIS ANTIBIÓTICA, restringiendo los mismos solo con infecciones documentadas o signos de sepsis.

-Realizar escala de valoración SCORTEN y valoración de SCD.

-Valoración ESTADO GENERAL Y OXIGENACIÓN por si fuera necesario intubación y respiración mecánica. -Realizar PRUEBAS LABORATORIO (hemograma, bioquímica, coagulación, pruebas de función hepática, IgA sérica), Rx tórax.

-CANALIZACIÓN DE SONDA VESICAL Y ACCESO VENOSO (si SCD elevada, canalizar via central) en áreas

de piel no involucradas.

-Búsqueda de FOCOS INFECCIOSOS, toma de muestras microbiológicas en mucosa nasal y rectal.

-Iniciar ANALGESIA Y SOLUCIONES PARENTERALES ( $2 \mathrm{ml} / \mathrm{kg} / \% \mathrm{SCD}$, Ringer Lactato) con control de liquidos, electrolitos, y constantes horarios.

-Administración profiláctica de ENOXAPARINA sc $40 \mathrm{mg} / 24 \mathrm{~h}$

-Cursar HOJAS DE INTERCONSULTA para Oftalmolología, Dermatología y Nutrición.

Valorar:

-DIETA ORAL

hiperproteica.

-SNG, si

afectación

mucosa oral.

-Complementar

con NTP, si

precisa

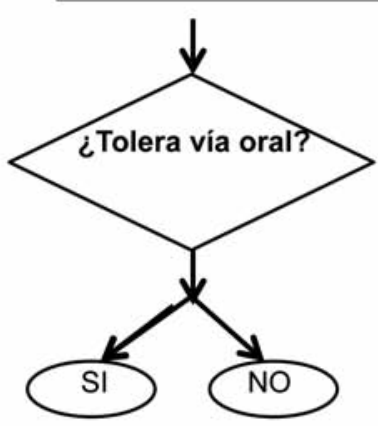

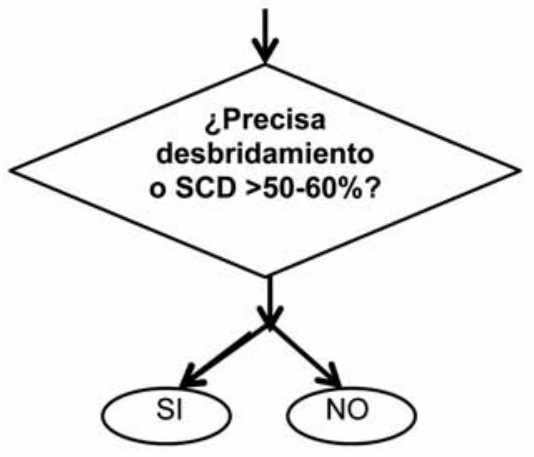

DESBRIDAMIENTO QUIRÚRGICO y la colocación de láminas de Biobrane $\odot$, con revisión diaria

CURAS DIARIAS ASEPTICAS con tul graso, desbridante y mupirocina tópicas y cambio de

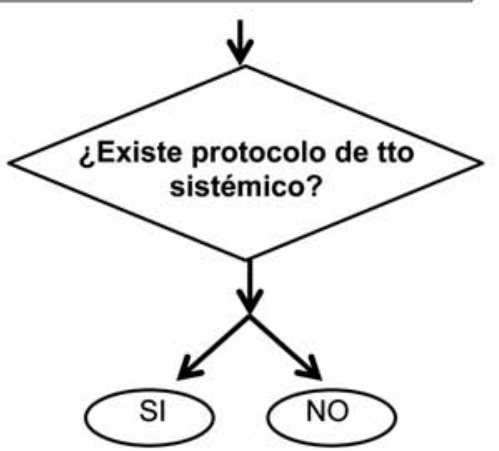
antibiótico tópico según cultivos de piel

\section{CUIDADOS DIARIOS}

-Cuidado oral con soluciones con clorexidina y/o antifúngicos, e hidratación de labios.

-Rehabilitación diaria ara preservar la movilidad de las extremidades.

-Mantener una habitación cálida para evitar la hipotermia.

-Evitar la manipulación innecesaria de la piel. El material adhesivo no deberá aplicarse directamente en la piel cuando sea posible.

-Exploración diaria de sacos conjuntivales y conjuntiva: lubricantes libres de conservantes y antibiótico tópico según Oftalmología.

-Vigilancia microbiológica estrecha con toma de cultivos de zonas desepidermizadas de forma habitual.

-Manipulación de medios invasivos con extrema asepsia: impregnación de las entradas de los catéteres centrales con antisépticos tipo clorhexidina.

-En los primeros dias, tratar fiebre secundaria a NET con paracetamol iv.

-Si sospecha de bacteriemia, hemocultivos, urocultivos y tratamiento antibiótico empírico protocolizado de cada unidad de quemados. 
- Retirada temprana del fármaco sospechoso.

- Enfoque multidisciplinario, con la participación fundamental de los Servicios de Dermatología, Oftalmología y Nutrición.

- Reanimación del paciente con fluidos tipo Ringer lactato de acuerdo con la fórmula de $2 \mathrm{ml} / \mathrm{kg} / \%$ SCD y ajuste según diuresis, sobre todo en las primeras semanas de ingreso del paciente.

- Ingreso en una Unidad de Quemados o en una Unidad de Cuidados Intensivos para seguimiento exhaustivo de la estabilidad hemodinámica del paciente, de su proceso de epitelización y para control infeccioso durante la evolución.

- Control de la temperatura de la habitación por el gran riesgo de estos pacientes de sufrir hipotermia.

- Cuidado de las heridas con asepsia estricta, siendo opcional la utilización de coberturas sintéticas o biológicas.

- Uso de antibióticos sólo cuando la infección esté demostrada con cultivos y por la sintomatología.

- Evitar el uso de corticoides, tanto previos como al ingreso.

- Minimización de las entradas invasivas tipo catéteres, y manipulación aséptica estricta de las mismas cuando sean necesarias.

- En lo posible utilizar nutrición enteral, dejando la parenteral como apoyo o en caso de intolerancia oral.

- Tratamiento adecuado del dolor con analgesia.

- Seguimiento y tratamiento exhaustivo de las lesiones oculares y de otras mucosas.

\section{Conclusiones}

La NET y el síndrome de superposición NET/SSJ son verdaderas urgencias vitales que requieren atención por equipos multidisciplinares, siendo la Unidad de Quemados, por su experiencia en el abordaje de grandes superficies desepidermizadas, el lugar idóneo para llevar a cabo este tratamiento.

Cualquier fármaco puede estar potencialmente implicado en la etiología de la NET, siendo los más frecuentes los AINES, anticomiciales, antibióticos, antirretrovirales y el alopurinol.

Los principales factores pronósticos de evolución de la enfermedad son el ingreso temprano en una unidad especializada, la retirada inmediata de los fármacos sospechosos, evitar la utilización de tratamientos previos al ingreso que no hayan demostrado eficacia, como los corticoides sistémicos o los antibióticos profilácticos, y el control de las enfermedades concomitantes, sobre todo de la presencia de procesos sépticos durante el ingreso.

Los tratamientos farmacológicos para esta enfermedad todavía no tienen un papel convincente, por lo que son necesarias más investigaciones futuras con estudios prospectivos aleatorizados multicéntricos que permitan sacar conclusiones y establecer protocolos estándar.

\section{Dirección del autor}

Dr. Felipe Castillo Muñoz

Av. Ansaldo 4, bloque Z, escalera 2, $8^{\circ} \mathrm{D}$

03540 Alicante, España.

e-mail: dr.felipecastillo@gmail.com

\section{Bibliografía}

1. Bastuji-Garin S, Rzany B, Stern RS, et al.: Clinical classification of cases of toxic epidermal necrolysis, Stevens-Johnson syndrome, and erythema multiforme. Arch. Dermatol. 1993; 129: 92-96.

2. Chave TA, Mortimer NJ, Sladden MJ et al.: Toxic epidermal necrolysis: current evidence, practical management and future directions. Br. J. Dermatol. 2005; 153: 241-253.

3. Bastuji-Garin S, Fouchard N, Bertocchi M, et al.: SCORTEN: Severity of illness score for toxic epidermal necrolisys. J. Invest. Dermatol. 2000; 115:149-153.

4. Ferrándiz-Pulido C, García-Fernández D, GómezMorell P, et al.: Síndrome de Stevens-Johnson y necrólisis epidérmica tóxica: revisión de la experiencia clínica en un Hospital Universitario (1989-2008). Med. Clin. (Barc). 2011 ; 136: 583-587.

5. Weinand $\mathbf{C}, \mathbf{X u ~ W}$, Perbix W, et al.: 27 years of a single burn centre experience with Stevens-Johnson syndrome and toxic epidermal necrolysis: analysis of mortality risk for causative agents. Burns. 2013; 39: 1449-1455.

6. Roujeau JC, Kelly JC, Naldi L, et al.: Medication use and the risk of Stevens-Johnson syndrome and toxic epidermal necrolysis. N. Engl. J. Med. 1995; 333: 16001607.

7. McGee T, Munster A.: Toxic epidermal necrolysis syndrome: mortality rate reduced with early referral to regional burncenter. Plast. Reconstr. Surg. 1998; 102: 1018-1022.

8. Sassolas B, Haddad C, Mockenhaupt M, et al.: ALDEN, an algorithm for assessment of drug causality in Stevens-Johnson syndrome and toxic epidermal necrolysis: comparison with case-control analysis. Clin. Pharm. Ther. 2010; 88: 60-68.

9. Zajicek R, Pintar D, Broz L, et al.: Toxic epidermal necrolysis and Stevens-Johnson syndrome at the Prague Burn Centre 1998-2008. J Eur Acad Dermatol Venereol. 2012; 26: 639-643

10. Schneck J, Fagot J, Sekula P, et al.: Effects of treatments on the mortality of Stevens Johnson syndrome and toxic epidermal necrolysis: a retrospective study on patients included in the prospective EuroSCAR Study. $J$. Am. Acad. Dermatol 2008; 58: 33-40.

11. Mockenhaupt M, Viboud C, Dunant A, et al.: StevensJohnson syndrome and toxic epidermal necrolysis: assessment of medication risks with emphasis on recently marketed drugs. The Euro-SCAR-study. J. Invest. Dermatol. 2008; 128: 35-44.

12. Downey A, Jackson C, Harun N, Cooper A.: Toxic epidermal necrolysis: review of pathogenesis and management. J. Am. Acad. Dermatol. 2012; 66: 995-1003.

13. French LE, Prins C.: Toxic epidermal necrolysis. In: Bolognia JL, Jorizzo JL, Rapini RP, editors. Dermatology. Edinburgh: Mosby; 2003. pp. 323-331. 
14. Shiga S, Cartotto R.: What are the fluid requirements in toxic epidermal necrolysis? J. Burn Care Res. 2010; 31: 100-104.

15. Ruiz-Maldonado R.: Acute disseminated epidermal necrolysis types 1,2 and 3: study of 60 cases. J. Am. Acad. Dermatol. 1985; 13: 623-635.

16. Kardaun SH, Jonkman MF.: Dexamethasone pulse therapy for Stevens-Johnson syndrome/toxic epidermal necrolysis. Acta Derm.Venereol. 2007;87:144-148.

17. Al-Mutairi N, Arun J, Osama NE, et al.: Prospective, non comparative open study from Kuwait of the role of intravenous immunoglobulin in the treatment of toxic epidermal necrolysis. Int. J. Dermatol. 2004; 43: 847851.

18. Brown KM, Silver GM, Halerz M, et al: Toxic epidermal necrolysis: does immunoglobulin make a difference? J. Burn. Care. Rehabil. 2004; 25: 81-88.

19. Huang YC, Li YC, Chen TJ.: The efficacy of intravenous immunoglobulin for the treatment of toxic epidermal necrolysis: a systemic review and meta-analysis. $\mathrm{Br}$. J. Dermatol. 2012; 167: 424-432.

20. Zhu QY, Ma L, Luo XQ, et al.: Toxic epidermal necrolysis: performance of SCORTEN and the score-based comparison of the efficacy of corticosteroid therapy and intravenous immunoglobulin combined therapy in China. J. Burn Care Res. 2012; 33: e295-308.

21. Narita YM, Hirahara K, Mizukawa Y, Kano Y, Shiohara T.: Efficacy of plasmapheresis for the treatment of severe toxic epidermal necrolysis: is cytokine expression analysis useful in predicting its therapeutic efficacy? $J$. Dermatol. 2011; 38: 236-245.

22. Kreft B, Wohlrab J, Bramsiepe I, et al: .Etoricoxibinduced toxic epidermal necrolysis: successful treatment with infliximab. J. Dermatol. 2010; 37: 904-906.
23. Valeyrie-Allanore L, Wolkenstein P, Brochard L, et al.: Open trial of ciclosporin treatment for Stevens-Johnson syndrome and toxic epidermal necrolysis. $\mathrm{Br}$. J. Dermatol. 2010; 163: 847-853.

24. Rajaratnamn R, Mann C Balasubramaniam P, et al.: Toxic epidermal necrolysis: retrospective analysis of 21 consecutive cases managed at a tertiary centre. Clin. Exp. Dermatol. 2010; 35: 853-862.

25. Ramos GE, Bolgiani AN, Patino O, et al.: Catheter infection risk related to the distance between insertion site and burned area. J. Burn Care Rehabil. 2001; 23: 266271.

26. Casey AL, Mermel LA, Nightingale P, et al.: Antimicrobial central venous catheters in adults: a systematic review and meta-analysis. Lancet Infect. Dis. 2008; 8: 763-776.

27. Palmieri TL, Greenhalgh DG, Saffle JR, et al.: A multicenter review of toxic epidermal necrolysis treated in U.S. burn centers at the end of the twentieth century. $J$. Burn Care Rehabil. 2002; 23: 87-96.

28. Boorboor P, Vogt PM, Bechara FG, et al.: Toxic epidermal necrolysis: use of Biobrane or skin coverage reduces pain, improves mobilisation and decreases infection in elderly patients. Burns. 2008; 34: 487-492.

29. Haber J, Hopman W, Gomez M, et al: .Late outcomes in adult survivors of toxic epidermal necrolysis after treatment in a burn center. J Burn Care Rehabil. 2005; 26:33-41.

30. Melloni Magnelli, L. ,Padrón Flores, A.E, Larrazábal Aguerrevere, L.I, Sony Avendaño, B.N. Necrolisis epidérmica tóxica inducida por fármacos. Caso clínico, Cir.plást.iberolatinoam. 2008, 34 (4): 305-312. 